\title{
A real-time editing method for multi-layered translucent materials
}

\author{
Hoe Min KIM*, Seung Joo LEE** and Kwan H. LEE*** \\ * Korea Photonics Technology Institute (KOPTI) \\ 9 Cheomdanbencheo-ro 108, Buk-Gu, Gwangju, 500-712, Korea \\ ** Korea Electronics Technology Institute (KETI) \\ 226 Cheomdangwagi-ro, Buk-Gu, Gwangju, 500-712, Korea \\ *** Gwangju Institute of Science and Technology (GIST) \\ 1 Oryong-Dong, Buk-Gu, Gwangju, 500-712, Korea \\ E-mail: sf2952@gist.ac.kr
}

\section{Received 6 November 2013}

\begin{abstract}
In this paper we introduce a practical method to evaluate the reflectance of a layered turbid medium with a thin layer, which is not possible with the previous methods based on the diffusion theory. To this end, we employ two different reflectance models simultaneously. One is the newly improved 2-flux model for a thin layer and the other is the diffusion model for a thick layer. To combine the evaluation results from these two different models, we introduce a re-parameterization approach which makes these models compatible with each other. We verify that the proposed method yields robust performance by comparing with the Monte Carlo simulation. We also validate visual results and comparing the rendering time ours with other models.
\end{abstract}

Key words : BSSRDF, Multi-layered translucent material, Realistic rendering, Real-time rendering

\section{Introduction}

At the present day, the aesthetic factors of a product that is influenced by geometric shape and surface appearance have had considerable implications for the product purchase of customers. With the reverse engineering (RE) that creates a $3 \mathrm{D}$ virtual model from a physical object, the rendering technique that realistically represents the object in a virtual space greatly helps the appearance design that determines the esthetic of the product and facilitates various product designs without manufacture of a mock-up. These techniques are able to reduce the development cost in early stage of product development.

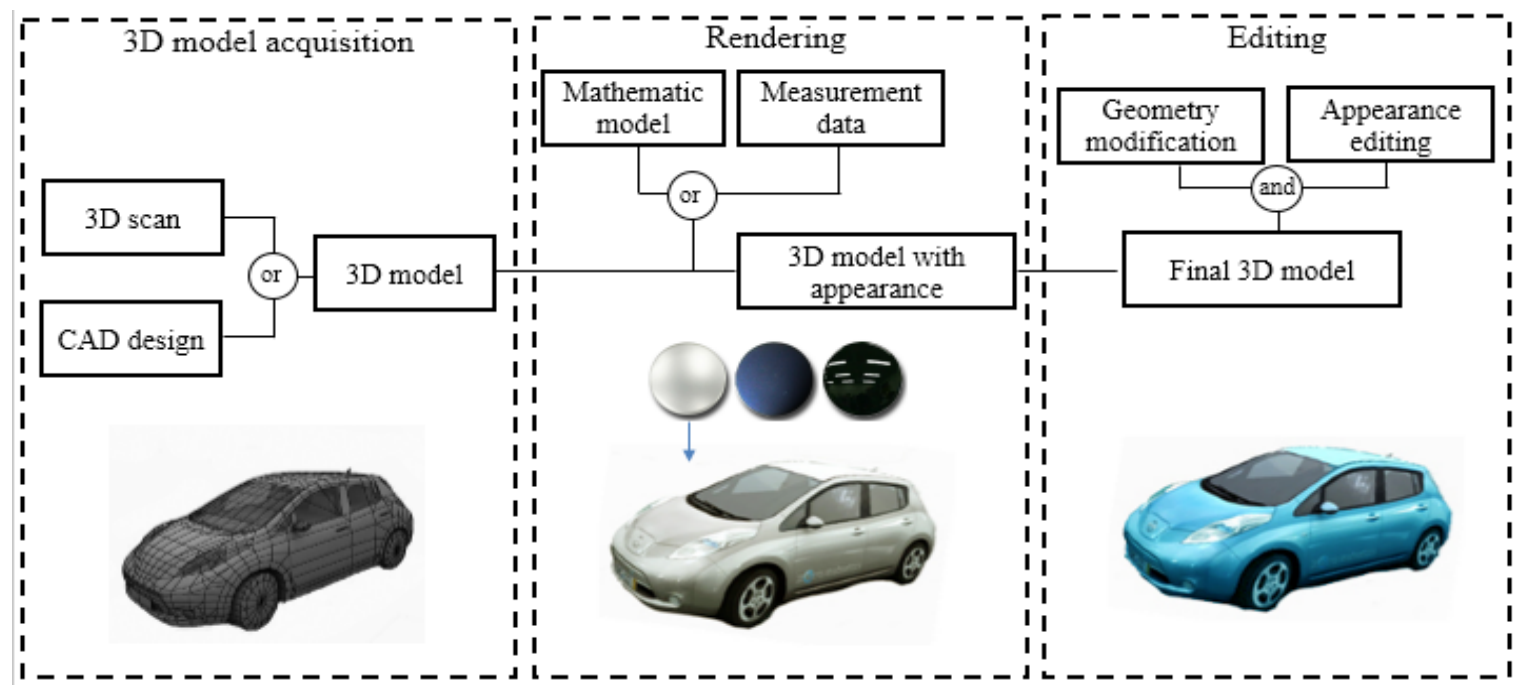

Fig. 1 The overall procedure in a $3 \mathrm{D} \mathrm{CAD}$ 
Fig. 1 describes the overall procedure showing how the geometry and surface appearance of a 3D CAD model in a design step is progress before developing the product. As shown in the figure, a 3D geometry is able to be created through a 3D scanner or CAD tool and an surface appearance on the $3 \mathrm{D}$ geometry produced by mathematic modeing or measurement data for optical properties of real materials. The measurement data detecting light transfort in real materials plays a leading role to realistically represent the 3D model, compared to the mathematic modeing such as Phong shading which is difficult to accurately represnet various appearances. The development for the acquisition method and data fitting algorithm which is suitable to meterial property is very important, since most of industrial products such as electrical goods and mechanical products is consists of complex materials including plastics, fiber, metal etc.

The object appearance description can be divided two main approaches depending on the surface reflectance propoerty. A bidirectional reflectance distribution function(BRDF) has to be used to represent opacity meterals such as metal, but it can not estimate subsurface scattering of light which is one of properties regarding translucent materials. To represent the translucent materials such as plastic and rubber, more complex reflectance funtion has to be developed. Fig 2. shows a electrical goods consisted of translucent(rubber) and opacity(metal) materials and describes optical property reflected in each materials.

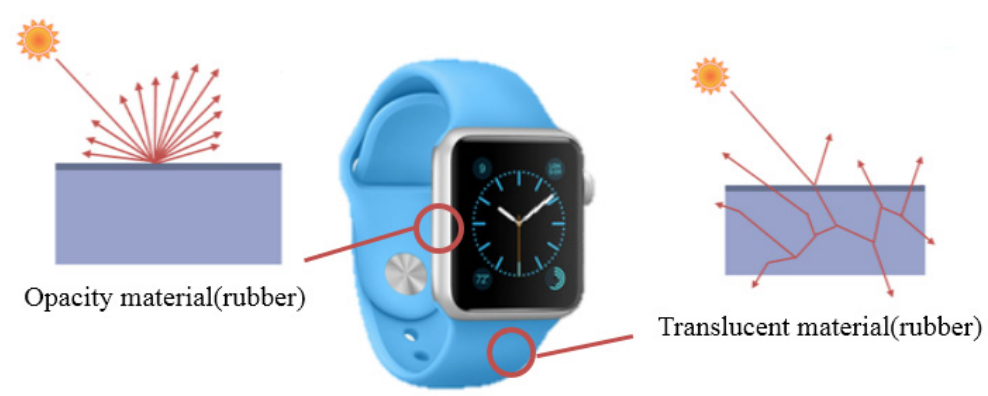

Fig. 2 The most of industrial products is consist of complex materials including plastic, rubber, and metal etc.

In the last stage of a product design, geometric modification and surface appearance editing can be performed according to the development strategy. However, it is difficult to edit translucent materials in real-time since the amount of computation is large. In this paper, we focus on the representation of translucent materials and propose a practical method to evaluate the reflectance of a layered turbid medium with a thin layer. We also propose novel method which can edit translucent materials in real-time.

Surface appearance can be represented by the optical analysis of incident light on a surface and its reflected light. Especially, translucent material that is important component of product appearance can be described by the analysis of scattering and absorption of the light in a subsurface of the object. The subsurface scattering effect is a unique phenomenon observed for translucent materials when the incident light emerges out of a different place from a point where it comes in. Many of the objects in our life exhibit translucent appearance and some of them have multi-layered structures. Based on a diffusion theory, the subsurface scattering of a translucent material can be described as:

$$
\mathrm{L}_{\mathrm{o}}\left(\mathrm{x}_{\mathrm{o}}, \omega_{\mathrm{o}}\right)=\frac{1}{\pi} \mathrm{F}_{\mathrm{t}}\left(\eta, \omega_{o}\right) \int_{\mathrm{A}} \mathrm{R}(\mathrm{r}) \mathrm{E}\left(\mathrm{x}_{\mathrm{i}}\right) \mathrm{dA}\left(\mathrm{x}_{\mathrm{i}}\right),
$$

where $r$ is the radial distance $\left(\left\|\mathrm{x}_{\mathrm{o}}-\mathrm{x}_{\mathrm{i}}\right\|\right)$ between the incident and exit points, $R(r)$ the diffusion equation describing the attenuation degree according to the distance $(r)$, and the rest of the notations of Eq. 1 are listed in Table 1. As you can see from Eq. 1, in order to estimate the outgoing radiance at a point, a number of irradiance samples on the object surface are required. Furthermore, to predict the light propagation in multi-layered translucent materials, we additionally need to take the inter-reflections between the individual layers into account.

Consequently, due to its complex light scattering process, the rendering of the multi-layered translucent material has been a challenging work. In this work we develop a new multi-layered translucent material editing system which enables us to change the various optical properties of the materials in real time. Fig. 1 shows the rendering pipeline for our system. We first obtain the diffusion profiles of each layer based on an existing diffusion approximation (Jensen, et al., 
2001)(Donner and Jensen, 2006) and minimize the time for the sampling process by a GPU. And then, we obtain the multi-layered diffusion profile by means of the QFHT, and find the wavelet coefficients by applying a non-linear wavelet analysis to the diffusion profile. Aside from this step, during the pre-computation process, we compute light transports corresponding to each wavelet basis. Lastly, we render an image by performing the dot product of the wavelet coefficients and the pre-computed light transports of each wavelet basis. Under the fixed lighting condition, we can achieve about 60 and 20 frames per second rendering performance for single and multiple layered materials, respectively.

Table 1 Notations for Ep. 1

\begin{tabular}{cl}
\hline \hline$x_{i}, x_{o}$ & Incident and outgoing points \\
$\omega_{i}, \omega_{o}$ & Incident and outgoing light directions \\
$L_{o}$ & Outgoing radiance \\
$E$ & Irradiance from each point \\
$F_{t}$ & Diffuse Fresnel transmittance \\
$\eta$ & Relative refractive index \\
\hline \hline
\end{tabular}

The rest of this paper is organized as follows: First, we refer the related work such as the rendering methods for multi-layered translucent materials and various material editing systems in section 2 . In section 3 and 4 , we discuss an existing method to generate the diffusion profile of the multi-layered translucent materials and describe the proposed method that significantly accelerates the previous method. In next section, we show that the diffusion profiles can be well approximated with a sum of wavelet basis, and explain the implementation of our translucent material editing system in section 6. Finally, we demonstrate rendering results of the proposed method and its performance.

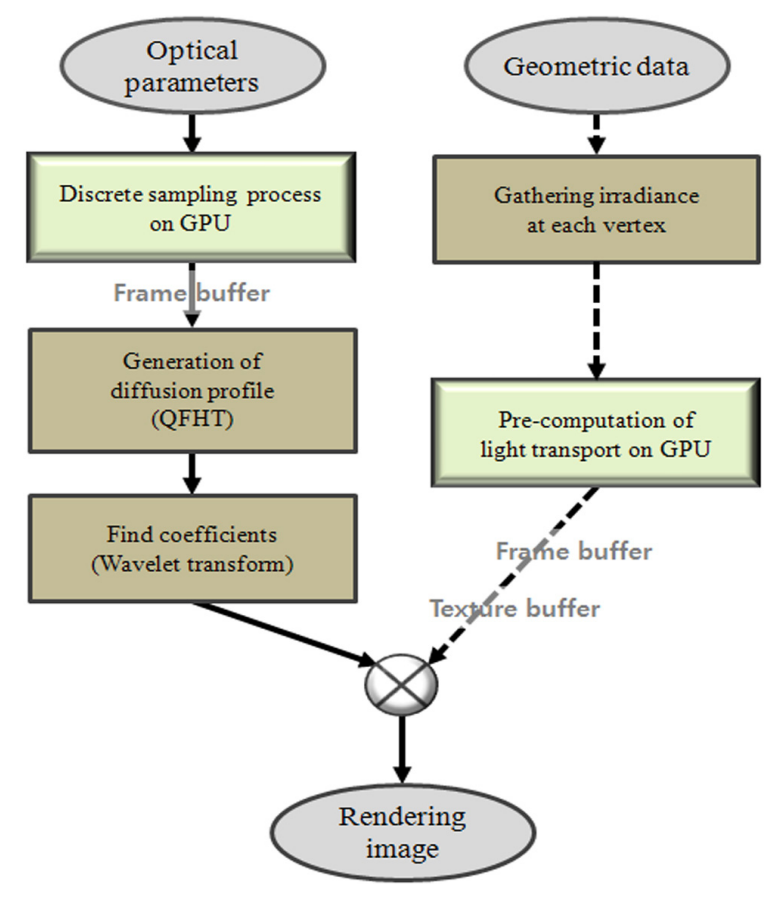

Fig. 3 Rendering pipeline for multi-layered translucent material editing. Solid line means the process for generating the diffusion profile of multi-layered translucent materials. Dash line is the pre-computation process for light transport. 


\section{Related work}

Light transport in a translucent material can be described by the Bi-directional Surface Scattering Reflectance Distribution Function (BSSRDF) (Nicodemus, et al., 1997). However, since it is an 8 degree equation, it requires a considerable computation time even with a modern computer system. Fortunately, a practical BSSRDF model for homogeneous translucent materials was introduced by Jensen et al. (Jensen, et al., 2001), who dramatically reduces the computation time by approximating the scattering model while maintaining the accuracy. This model was then combined with a two-hierarchical integration technique (Jensen and Buhler, 2002) so as to further accelerate the rendering process of translucent materials. Since then, it has become a popular model to render translucent objects in computer graphics.

\subsection{Multi-layered translucent material}

Hanrahan and Krueger (Hanrahan and Krueger, 1993) proposed a subsurface scattering model for layered materials by deriving an first-order analytical solution for single scattering events. However, this model cannot accurately describe the reflectance of translucent materials due to its generality.

Donner and Jensen (Donner and Jensen, 2005) introduced a novel method to efficiently convolve the diffusion profiles of the individual layers of a multi-layered material. Rather than performing complex convolution computation in a space domain they converted the complex convolution operation into a simple multiplication by applying 2D Fourier transform. Although this method enables us to obtain the diffusion profile of the multi-layered material much faster compared to a naive convolution approach, it still takes several seconds to obtain the diffusion profile.

d'Eon et al. (d'Eon, et al., 2007) developed a real-time rendering system which makes it possible to reproduce multilayered materials realistically like human skin under a dynamic lighting condition.

Based on the fact that a convolution of the polynomials consisting of a set of Gaussians can be easily computed in a frequency domain, they fitted a set of Gaussians to the individual diffusion profiles in advance and combined the Gaussians at run-time using a GPU. However, because this method requires going through the fitting process whenever the shape of the diffusion profile changes, it is not suitable for our fast material editing applications based on the optical parameters.

A versatile BSSRDF model (d'Eon and Irving, 2011) has also appeared to cover a broad range of homogeneous translucent materials, which have been impossible with the existing reflectance models. This model deals with not only optically thin translucent materials but also multi-layered materials. However, this model still has inherited the fitting process from the method by d'Eon et al. (d'Eon, et al., 2007), so that it cannot change the optically parameters of translucent materials.

\subsection{Material editing}

Compared to the researches for opaque materials (Ben-Artzi, et al. 2006)(Colbert, et al., 2006)( Ben-Artzi, et al., 2008), researches for editing translucent materials have relatively less progressed. Xu et al. (Xu, et al, 2007) have shown that both single scattering attenuation function and multiple scattering diffusion profiles can be efficiently approximated with a small number of polynomial piecewise curves. But, it needs a cumbersome step to determine the appropriate locations of these piecewise curves in advance.

Wang et al. (Wang, et al., 2008) also developed a voxel based material editing system that manipulates both translucent materials and lighting conditions in real time. They converted the diffusion profile into twelve simple 1D functions through a data-driven approach using PCA analysis.

Song et al. (Song, et al., 2009) introduced a new method for editing heterogeneous translucent materials. By decoupling local point-based scattering profiles from a measured material samples, they developed a system which enables users to control the heterogeneous materials intuitively maintaining the visual consistency.

Unfortunately, however, none of these editing methods can be directly extended to deal with multi-layered translucent materials. In this work we focuses on developing a real-time editing system for multi-layered translucent materials by building up existing methods by $\mathrm{Xu}$ et al. (Xu, et al, 2007) and Donner and Jensen (Donner and Jensen, 2005). We significantly reduce the computation time for the convolution process for the diffusion profiles by using the QFHT. We also develop a robust real-time BSSRDF editing method by introducing analytic wavelet decomposition method. 


\section{Diffusion profile of a multi-layered translucent material}

The absolute diffusion equation $(R(r))$ in Eq. 1 is represented by a sum of single scattering attenuation and multiple scattering diffusion terms. Since the highly scattering materials that we focus on this paper are mainly affected by the multiple scattering effect (Jensen and Buhler, 2002), we only deal with the multiple scattering in this paper. As mentioned, the diffusion profile due to the multiple scattering can be easily obtained based on the diffusion theory. Jensen et al. (Jensen, et al., 2001) generated the diffusion profiles of semi-infinite homogeneous translucent materials by the di-pole approximation. Donner and Jensen (Donner and Jensen, 2005) developed a novel method to efficiently derive the diffusion profiles of multi-layered translucent materials using both multi-pole approximation and Kubelka-Munk composing theory (Kubelka, 1954).

The reflectance diffusion profile due to 2-layered materials can be formulated by complex and infinite expansions including a huge number of convolution operations as:

$$
\mathrm{R}(\mathrm{r})=\mathrm{R}_{\mathrm{u}}^{+}+\mathrm{T}_{\mathrm{u}}^{-} * \mathrm{R}_{\mathrm{b}}^{+} * \mathrm{~T}_{\mathrm{u}}^{+}+\mathrm{T}_{\mathrm{u}}^{-} * \mathrm{R}_{\mathrm{b}}^{+} * \mathrm{R}_{\mathrm{u}}^{-} * \mathrm{R}_{\mathrm{b}}^{+} * \mathrm{~T}_{\mathrm{u}}^{+}+\mathrm{T}_{\mathrm{u}}^{-} * \mathrm{R}_{\mathrm{b}}^{+} * \mathrm{R}_{\mathrm{u}}^{-} * \mathrm{R}_{\mathrm{b}}^{+} * \mathrm{R}_{\mathrm{u}}^{-} * \mathrm{R}_{\mathrm{b}}^{+} * \mathrm{~T}_{\mathrm{u}}^{+}+\cdots
$$

Here, the superscriptions, + and -, denote the light direction with respect to the incoming light direction and the subscriptions, $u$ and $b$, are the upper and below layers, respectively. For simplicity, we leave out the variable, $r$, on the right side. It is worthy to note that a single layer can have two separate reflectance and transmittance profiles according to the light direction, because in most cases the relative refractive indices of the top and bottom layers are not identical.

Fortunately, based on the fact that Fourier transform of the convolution between two functions can be replaced by a simple multiplication of their individual Fourier transforms in the frequency domain, Eq. 2 can be converted into a concise expression including the Fourier transform as:

$$
\begin{aligned}
\mathrm{R}(\mathrm{r}) & =\mathrm{iFT}\left[\mathfrak{R}_{u}^{+}+\mathfrak{J}_{u}^{-} \mathfrak{R}_{b}^{+} \mathfrak{J}_{u}^{+}+\mathfrak{J}_{u}^{-} \mathfrak{R}_{b}^{+} \mathfrak{R}_{u}^{-} \mathfrak{R}_{b}^{+} \mathfrak{J}_{u}^{+}+\cdots\right] \\
& =\operatorname{iFT}\left[\mathfrak{R}_{u}^{+}+\frac{\mathfrak{J}_{u}^{-} \mathfrak{R}_{b}^{+} \mathfrak{I}_{u}^{+}}{1-\mathfrak{R}_{b}^{+} \mathfrak{R}_{u}^{-}}\right]
\end{aligned}
$$

where $\mathfrak{R}$ and $\mathfrak{I}$ are the Fourier transformed reflectance and transmittance profiles of each layer, respectively, and iFT means inverse Fourier transform. Similarly, we can also obtain a transmittance profile of multi-layered material. Although this methodology must be a much simpler approach than the conventional convolution one, it should be noted that we need five forward $\left(\mathfrak{R}_{u}^{+}, \mathfrak{I}_{u}^{-}, \mathfrak{I}_{u}^{+}, \mathfrak{R}_{u}^{-}, \mathfrak{R}_{b}^{+}\right)$and one inverse transformation $(i F T)$ even for a single diffusion reflectance profile of a multi-layered translucent material.

\section{$4 \quad$ Fast generation of diffusion profile}

In the previous section we explained the existing method to obtain the diffusion profiles of multi-layered translucent materials. In this section we concentrate on how to reduce the time for obtaining the diffusion profiles significantly. To generate a discrete diffusion profile, we extract 4,096 samples per diffusion curve. Such a sampling density ensures the accurate reconstruction for the diffusion profile including high frequency and is a sufficiently enough density to prohibit errors which could occur during the frequency transform step. For a discrete diffusion profile of a semi-finite homogeneous translucent material, the di-pole computations are required as the number of samples. On the other hands, for a diffusion profile of the finite translucent material lots of multi-pole computations are necessary. Furthermore, in order to obtain the diffusion profiles of multi-layered materials, 4,096 di-pole computations for the below layer( $\left.R_{b}^{+}\right)$and $16,384(4 \times$ 4,096) multi-pole computations for the upper layer $\left(\mathrm{R}_{\mathrm{u}}^{+}, \mathrm{T}_{\mathrm{u}}^{-}, \mathrm{T}_{\mathrm{u}}^{+}, \mathrm{R}_{\mathrm{u}}^{-}\right.$) are required.

Therefore, we adopt the latest work (Kim, et al., 2011) for fast convolution computation so as to rapidly generate the diffusion profiles of multi-layered translucent materials. As mentioned

before, because a huge number of similar computations for the diffusion equations are repeated, the time for sampling process should not be ignored.

As referred in (Kim, et al., 2011), the sampling process could need more time than a frequency analysis step. Thus, we also take advantage of a parallel computing methodology using a GPU. Based on the fact that the multi-pole diffusion equation includes many of the di-pole equations in itself, we perform the multi-pole computations for each sampling 
point using a shader program. The location information of each sample is stored in a texture such that it could be shared with all of the shader programs. A couple of fragment shaders are implemented to calculate the reflectance and transmittance for each location. The computation results are transmitted to system memory through a frame buffer so that they can be used to reconstruct individual diffusion profiles for each layer.

Despite such a fast sampling method using a parallel computing, it still takes several seconds to generate the diffusion profile of the multi-layered translucent material. It is due to the computation time for the convolution process to combine them. Fortunately, we can find a solution for this problem in the Quasi fast Hankel transform (QFHT) (Siegman, 1977).

For the convolution operations between diffusion profiles, 2D Fourier transform is required. Since the diffusion profiles are cylindrically symmetric, they can be managed with the zeroth order 1D Hankel transform as:

$$
\mathrm{g}(\rho)=2 \pi \int_{0}^{\infty} \mathrm{rf}(\mathrm{r}) \mathrm{J}_{0}(2 \pi \rho \mathrm{r}) \mathrm{dr}
$$

where $\mathrm{f}(\mathrm{r})$ is an input profile, $\mathrm{g}(\rho)$ the transformed $\mathrm{f}(\mathrm{r})$, and $\mathrm{J}_{0}$ is a zeroth order Bessel function of the first kind. Nevertheless, because the Hankel transform includes Bessel function in it, it still needs considerable computation time. Siegman (Siegman, 1977). found that the Hankel transform problem can be converted into the fast Fourier transform problem by using exponentially increasing non-linear sampling method and Fourier cross-correlation theorem. If we replace $\mathrm{r}$ and $\rho$ in Eq. 4 with $\mathrm{r}_{0} \mathrm{e}^{\alpha \mathrm{x}}$ and $\rho_{0} \mathrm{e}^{\alpha \mathrm{y}}$, where $\mathrm{r}_{0}, \rho_{0}$, and $\alpha$ are arbitrary values enabling to extract samples sufficiently according to the range of the diffusion curve and the given number of samples, it can be changed to:

$$
\hat{g}(y)=\int_{-\infty}^{\infty} \hat{f}(x) \hat{j}(x+y) d x
$$

Here, where $\hat{\mathrm{g}}(\mathrm{y})=\rho \mathrm{g}(\rho), \hat{\mathrm{f}}(\mathrm{x})=\mathrm{rf}(\mathrm{r})$ and $\hat{\mathrm{j}}(\mathrm{x}+\mathrm{y})=2 \pi \alpha \mathrm{r} \rho \mathrm{J}_{0}(2 \pi \mathrm{r} \rho)$. Then, according to Fourier cross-correlation theorem, Eq. 5 can be expressed as Eq. 6, which can be numerically evaluated by the discrete fast Fourier transform(DFFT).

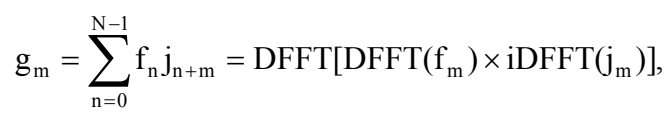

where $g_{m}, f_{n}$ and $j_{n+m}$ are exponentially sampled data from $\hat{g}(y), \hat{f}(x)$ and $\hat{j}(x+y)$. And DFFT and iDFFT mean the forward and inverse DFFT, respectively. Although the QFHT provides excellent computational efficiency, it often causes an error. Fortunately, this problem can be readily solved by making use of the lower-end correction algorithm (Sheng and Siegman, 1980) without affecting the rendering speed.

As can be seen in Fig. 2, the proposed convolution approach including the correction algorithm produces almost identical convolution results to the existing method (Donner and Jensen, 2005). Since the QFHT uses a non-uniform sampling strategy which extracts samples exponentially, we need an additional uniform sampling step for further process. However, it hardly causes errors because the diffusion profiles rapidly converge to zero and do not have any fluctuation in the shape.

In summary, we accelerate the sampling process by the parallel computing and minimize the computation time by applying the QFHT to convolve the individual diffusion profiles. The QFHT reduces the computational complexity of $\mathrm{N}^{2}$ into $\log _{2} 2 \mathrm{~N}$. This approach takes less than $50 \mathrm{~ms}$ to obtain the diffusion profiles of the multi-layered translucent materials for the whole RGB channels.

Recently, a noble method to obtain the diffusion profile of multi-layered translucent materials has been published (Liemert and Kienle, 2010). Nevertheless, compared to this method, the fast convolution technique (Kim, et al., 2011) employed in our algorithm has two representative advantages. First, the fast convolution method has a computational efficiency comparable to the other recent method even when it is executed in a low-priced system. Second, the convolution method is not restricted to the light transport theory which is used for generating diffusion profiles, e.g., di-pole and multi-pole theories. For example, since it can be used along with the other theories like $P N$ approximation (Lee, et al., 2010) suitable for weakly scattering materials, it can provide more accurate diffusion profiles for multi-layered materials including the weakly scattering materials. 


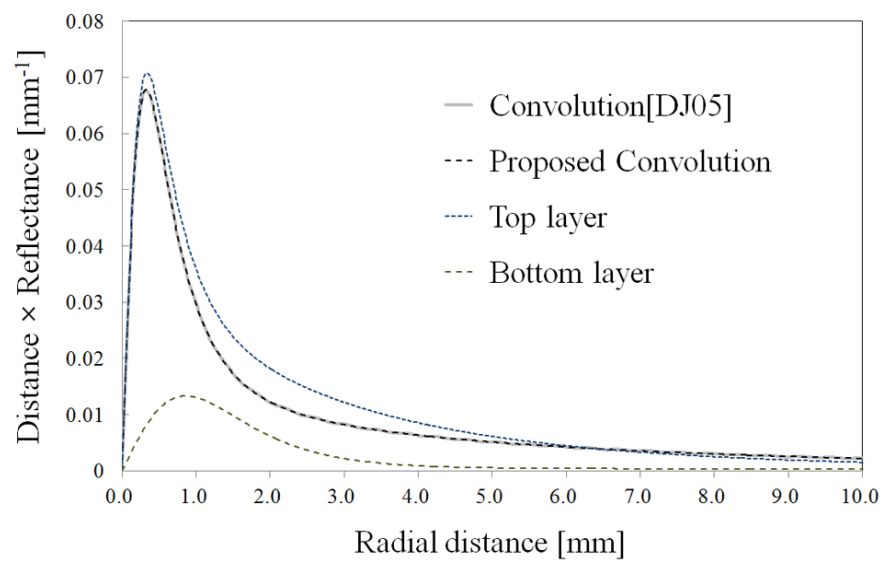

Fig. 4 Comparison of the convolution results between Donner and Jensen (Donner and Jensen, 2005) and the proposed convolution method. The materials used are marble(top) and skimmilk(bottom) (Jensen, et al., 2001). It shows that their difference is almost imperceptible.

\section{Acquisition of optical properties}

We use measurement system proposed by (Lee, et al., 2010) to acquire absorption and scattering properties in real translucent materials. Various translucent materials used in industrial products are detected by the measurement system. Fig. 5(top) shows the measurement system and translucent material samples used in this paper. To confirm the results of the measurement system, we also show the rendering results using the absorption and scattering properties acquired from the measurement system. Fig. 5(bottom) describes the rendering results using the optical properties of plastic and the results vary according to translucency.
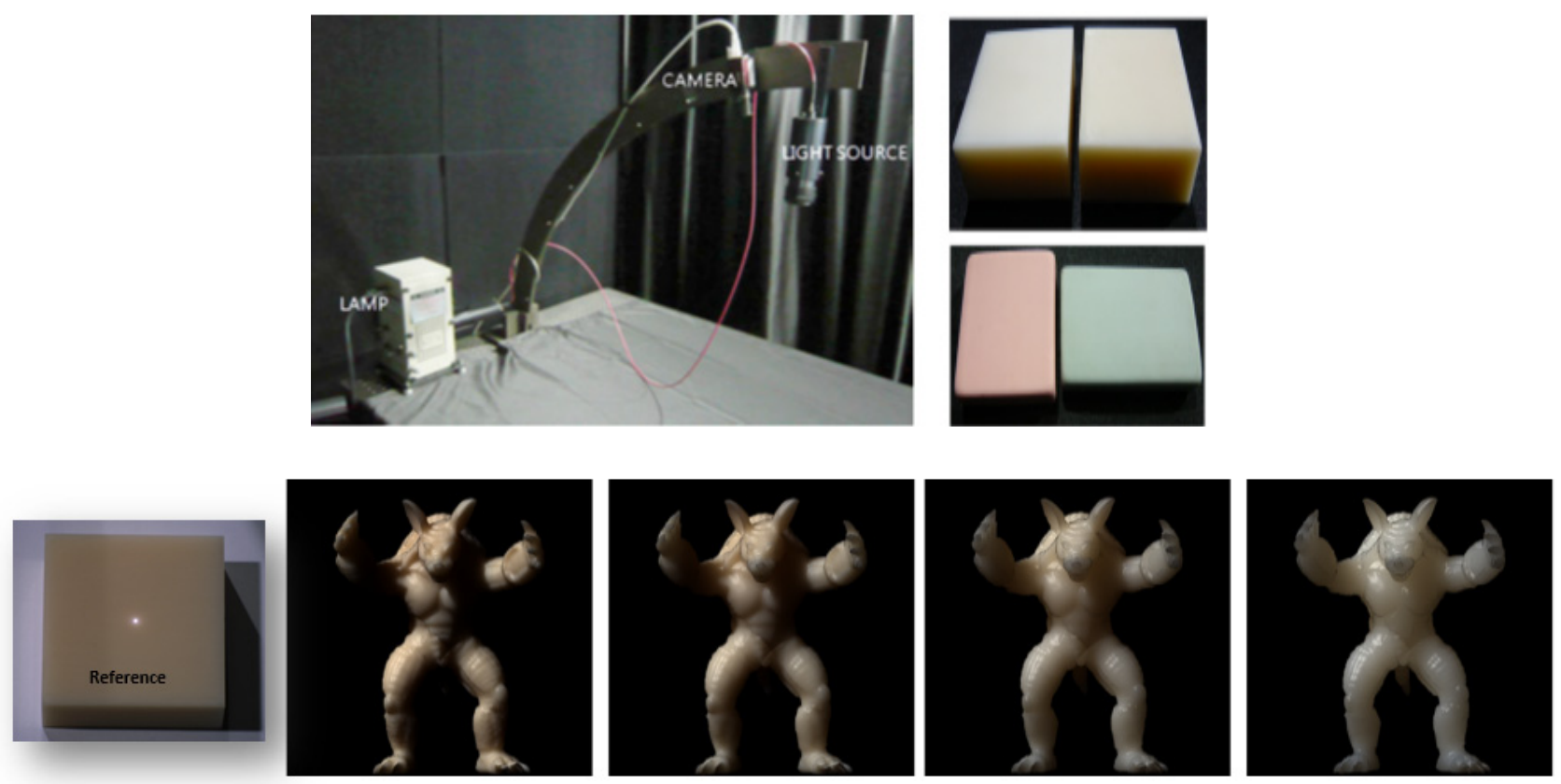

Fig. 5 The measurement system and translucent material samples(top) and rendering results using the optical properties of plastic(bottom). 


\section{Diffusion profile approximation}

A basic idea for our material editing method is quite similar to the pre-computed radiance transfer(PRT) approach for relighting applications. The existing PRT methods approximate the complex lighting with a linear combination of certain basis functions such as spherical harmonic (Sloan, et al., 2002) and wavelet $(\mathrm{Ng}$, et al., 2003). Likewise, the translucent material editing methods also approximate the diffusion profile with a sum of basis functions and pre-compute the subsurface light transports corresponding to each basis function to combine them at run-time for a rendering image.

d'Eon et al. (d'Eon, et al., 2007) employed Gaussian as the basis function and Wang et al. (Wang, et al., 2008) used an orthogonal matrix of PCA analysis. However, their methods require relatively long time to finding an optimal basis set.

On the other hand, $\mathrm{Xu}$ et al. (Xu, et al., 2007) demonstrated that the diffusion profile can be well approximated in real-time using a piecewise polynomial basis even though their method is also based on a numerical method. However, it needs an additional step to determine the optimal locations of the knots which link the polynomial basis. To this end, they adopted the average of the knots optimized for the three representative diffusion profiles as the initial knot locations. Therefore, the approximation accuracy of this approach depends greatly on the initial knots and it needs to find the optimal knot locations whenever the number of polynomial basis or the range of the optical parameters changes. Moreover, it could cause unstable approximation results, especially when applied to the multi-layered materials which are composed of several materials with different optical properties.

In this section, we present a robust approximation algorithm which is a fast analytical method and can be applied to a broader range of materials including multi-layered materials.

The Fourier transform is a widely used mathematical tool to approximate a given signal with a good efficiency. However, this transform method fails to reconstruct the diffusion curves by causing a fatal ringing artifact without sufficient high frequency data. It is due to that the diffusion profile is a kind of rapidly decaying signal whose energy abruptly decreases along with $x$-axis. Contrary to the Fourier transform, the wavelet analysis is capable of capturing the shape of the decaying curve much better than the Fourier transform due to its spatial analysis ability.

However, the conventional wavelet analysis based on low pass filter (LPF) still fails to reconstruct the original shape of a rapidly decaying curve like the diffusion profile. Therefore, we boost up the compression efficiency of the wavelet analysis by adaptively choosing the basis. In other words, we give a priority to the basis with high power instead of the low frequency basis. Because most of the light energy of the diffusion profiles concentrates near to the incident point, we utilize more wavelet basis located near the incident point than the conventional LPF based method when approximating the diffusion profile. In this paper, for the convenience, we call this approach the non-linear wavelet analysis. Fig. 6 shows that the proposed non-linear wavelet can well approximate the diffusion profiles compared to the conventional LPF based Haar wavelet.

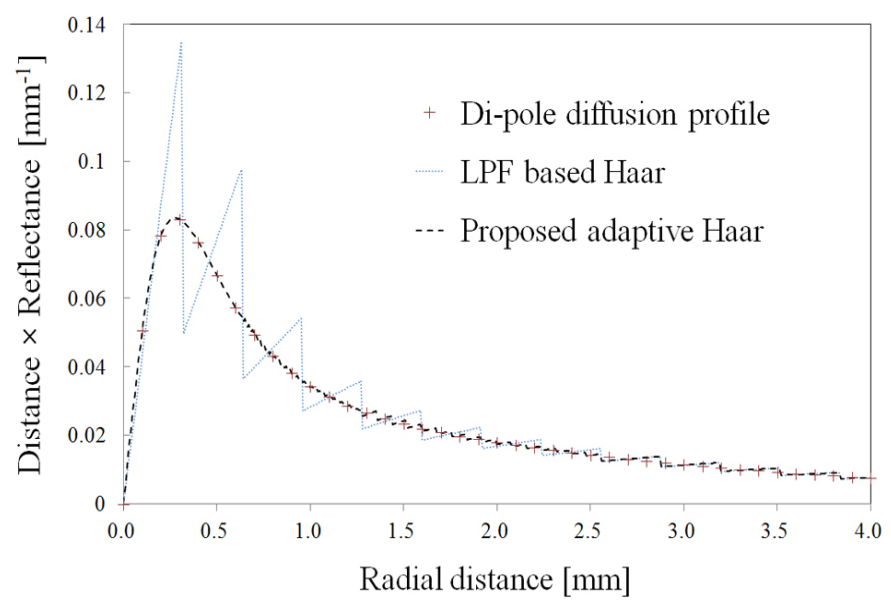

Fig. 6 Reconstruction results for the diffusion profile of marble(green channel) (Jensen, et al., 2001) with LPF based and proposed non-linear Haar wavelet. 
The non-linear wavelet has been first employed to build a real-time rendering system considering all-frequency illumination by $\mathrm{Ng}$ et al. ( $\mathrm{Ng}$, et al., 2003). It keeps large basis instead of low frequency ones from the illumination, thereby it is capable of representing both soft and sharp lighting effect at the same time with a small number of basis. When the non-linear wavelet is used for the illumination analysis, we are unable to predict the location of the largest basis because it greatly changes by the lighting environment. On the contrary, since the largest basis of the diffusion profile is concentrated on the front side of the profile, the diffusion profile approximation can be performed more efficiently than that for relighting applications by using a fixed small number of basis functions. Therefore, we apply the non-linear wavelet to approximate the diffusion profiles and it makes possible to rapidly find the wavelet coefficients corresponding to each basis. This method just takes less than $1 \mathrm{~ms}$ even to analyze three RGB diffusion profiles with 4,096 samples. In addition, we need not to concern about the locations of the knots unlike the method by (Xu, et al., 2007).

If we assume $R(r)$ is approximated with a sum of wavelet basis as:

$$
\mathrm{R}(\mathrm{r}) \approx \mathrm{c} \Phi(\mathrm{r})+\sum_{\mathrm{i}, \mathrm{j}} \mathrm{w}_{\mathrm{i}, \mathrm{j}} \Psi_{\mathrm{i}, \mathrm{j}}(\mathrm{r}), \quad j=\{1,2, \ldots, N\}, i=\left\{1,2, \ldots, 2^{j-1}\right\}
$$

Here, $\Phi(\mathrm{r})$ and $\Psi_{\mathrm{i}, \mathrm{j}}(\mathrm{r})$ denote scaling function and wavelet function, and $c$ and $\mathrm{w}_{\mathrm{i}, \mathrm{j}}$ are the coefficients for these functions. And $j$ and $i$ are the subscripts representing scaling and translation factors, respectively. It should be noted that there could be many translation factors per scaling factor. If we substitute Eq. 7 for $R(r)$ in Eq. 1, Eq. 1 can be rearranged as:

$$
\begin{aligned}
\mathrm{L}_{\mathrm{o}}\left(\mathrm{x}_{\mathrm{o}}, \omega_{\mathrm{o}}\right) & =\frac{1}{\pi} \mathrm{F}_{\mathrm{t}}\left(\eta, \omega_{o}\right) \int_{\mathrm{A}}\left[\mathrm{c} \Phi(\mathrm{r})+\sum_{\mathrm{i}, \mathrm{j}} \mathrm{w}_{\mathrm{i}, \mathrm{j}} \mathrm{\Psi}_{\mathrm{i}, \mathrm{j}}(\mathrm{r})\right] \mathrm{E}\left(\mathrm{x}_{\mathrm{i}}\right) \mathrm{dA}\left(\mathrm{x}_{\mathrm{i}}\right), \\
& =\frac{1}{\pi} \mathrm{F}_{\mathrm{t}}\left(\eta, \omega_{o}\right) \int_{\mathrm{A}}\left[\mathrm{cT} \mathrm{T}_{0}\left(\mathrm{x}_{\mathrm{o}}\right)+\sum_{\mathrm{i}, \mathrm{j}} \mathrm{w}_{\mathrm{i}, \mathrm{j}} \mathrm{T}_{\mathrm{i}, \mathrm{j}}\left(\mathrm{x}_{\mathrm{o}}\right)\right],
\end{aligned}
$$

where,

$$
\mathrm{T}_{0}=\int_{\mathrm{A}} \Phi E\left(\mathrm{x}_{\mathrm{i}}\right) \mathrm{dA}\left(\mathrm{x}_{\mathrm{i}}\right), \quad \mathrm{T}_{\mathrm{i}, \mathrm{j}}=\int_{A} \Psi_{i, j}(r) E\left(\mathrm{x}_{\mathrm{i}}\right) \mathrm{dA}\left(\mathrm{x}_{\mathrm{i}}\right) .
$$

In this paper, $T(x o)$ denotes transport function, which represents the radiance energy transferred by a certain basis function $(\Phi, \Psi)$ from irradiance on the whole surface of the object. As you can see from Eq. 8, if we pre-compute the light transport, we can quickly calculate the outgoing radiance by performing dot product of the pre-computed light $\operatorname{transports}(T(x o))$ and the corresponding wavelet coefficients $(c, w)$ at run-time. Fig. 7 shows the comparison of the RMS
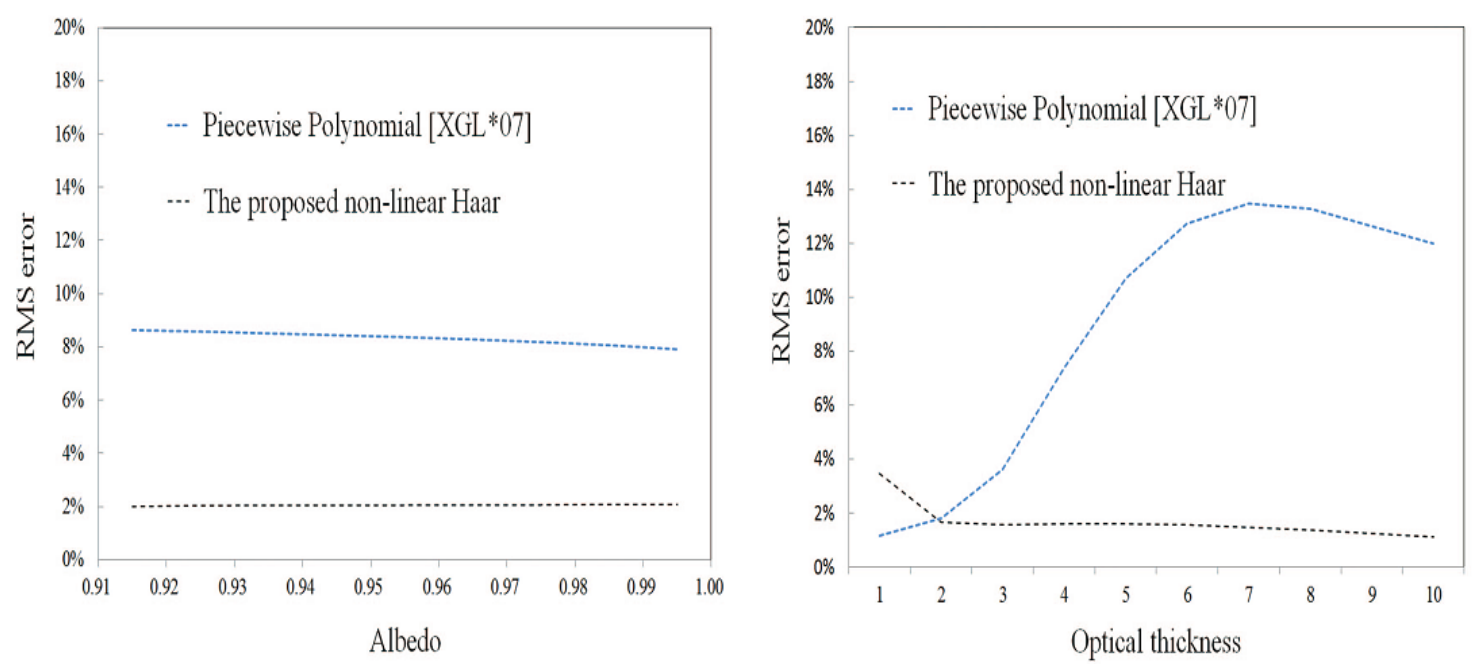

Fig. 7 Comparison of the approximation results by piecewise polynomial based method (Xu, et al., 2007) and the proposed non-linear based one with respect to the albedo(left) and optical thickness(right). 
error between our wavelet-based approximation method and the existing polynomial-based fitting approach (Xu, et al, 2007). Even though both methods yield stable approximation results regardless of the albedo of the materials, the proposed method introduces less error than the previous polynomial-based method (Xu, et al, 2007). On the contrary, it can also be noted that the approximation accuracy is strongly related to the optical thickness of the materials. And the proposed method provides the robust approximation results for a broad range of material except for the optically thin materials.

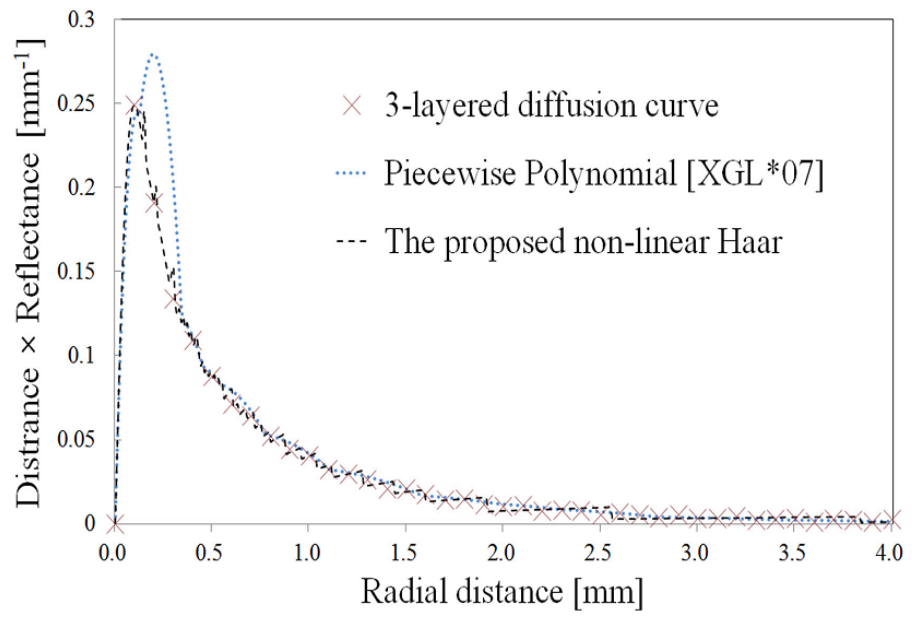

Fig. 8 Approximation results for a 3-layered translucent material by the previous piecewise polynomial based method (Xu, et al, 2007) and the proposed non-linear Haar based one. The 3-layered material consists of marble, potato and ketchup (Jensen, et al., 2001).

Fig. 8 compares the approximation accuracy for the diffusion profile of a 3-layered translucent materials. Since the light energy is concentrated on a region around the incidence point of the light, the approximation on the region(below Approx. 0.4) is important for the accurate prediction of the light propagation. An inaccurate approximation on the region can cause serious rendering errors such as spots, stripes and incorrect colors. Therefore, the proposed method focuses on a region around the incidence point of the light. The proposed method produces the successful result on the region as shown in Fig. 5. It can be seen that for the multi-layered materials with various different optical properties the proposed method gives the better approximation results than the previous method (Xu, et al, 2007).

The Daubechies can be a candidate to replace the Haar wavelet, but, according to our experiments, the Daubechies wavelet does not give as much satisfactory performance as expected. The approximation result using Haar wavelet does not depend on a shape of a given curve, but the approximation result by Daubechies wavelet can be changed by a shape of a given curve. We also confirm that Daubechies wavelet produces bad results when 32 or fewer bases are used. Furthermore, it requires very expensive computational cost by complex of the wavelet function. In this paper that emphasize on rendering speed, therefore, we choose the Haar wavelet as a basis function.

\section{$7 \quad$ Rendering}

From the previous two sections, we discussed how to obtain the diffusion profile more quickly and approximate the profile efficiently with a sum of wavelet basis. In this section, we explain the pre-computation step for the subsurface light transport and introduce the proposed editing system in detail.

\subsection{Pre-computation of radiance transport}

Since we assume a fixed illumination, we can pre-compute the subsurface light transports off-line so that we can readily take advantage of them at run-time. To this end, we first evaluate the irradiance $(E(x o))$ at each vertex by taking Fresnel term $(F t(\eta))$ on the object surface and the lighting condition into account. We then pre-compute the light 
$\operatorname{transports}(T(x o))$ associated with each wavelet based on the vertex irradiances and the geometric information of the whole vertices such as location and area. If we use 64 basis, we need 64 iterations for the entire pre-computation. To this end, we can build a hierarchical data structure for the rapid light transports evaluation using an octree (Jensen and Buhler, 2002), which makes it possible to evaluate each outgoing radiance without considering all the vertex irradiances. By using a solid angle in the octree as a criterion, we can adaptively choose the irradiance nodes which consist of a set of individual vertex irradiances. Even though a method to estimate the optimal solid angle has been proposed (Jensen and Buhler, 2002), it heavily depends on the lighting condition, wavelength as well as the target object shape.

Table 2 List of the wavelet basis used in our method. About 9,000 samples are tested(the sampling resolution is 0.01 about albedo, 0.1 about optical thickness, respectively)

\begin{tabular}{ccl}
\hline \hline Basis & No. of Basis & \multicolumn{1}{c}{ Coefficient for each basis } \\
\hline$\Psi_{X, 1}$ & 1 & $c$ \\
$\Psi_{X, 2}$ & 1 & $w_{1,1}$ \\
$\Psi_{X, 3}$ & 1 & $w_{1,2}$ \\
$\Psi_{X, 4}$ & 1 & $w_{1,3}$ \\
$\Psi_{X, 5}$ & 1 & $w_{1,4}$ \\
$\Psi_{X, 6}$ & 2 & $w_{1,5}, w_{2,5}$ \\
$\Psi_{X, 7}$ & 2 & $w_{1,6}, w_{2,6}$ \\
$\Psi_{X, 8}$ & 3 & $w_{1,7}, w_{2,7}, w_{3,7}$ \\
$\Psi_{X, 9}$ & 5 & $w_{1,8}, w_{2,8}, \cdots, w_{5,8}$ \\
$\Psi_{X, 10}$ & 7 & $w_{1,9}, w_{2,9}, \cdots, w_{7,9}$ \\
$\Psi_{X, 11}$ & 10 & $w_{1,10}, w_{2,10}, \cdots, w_{10,10}$ \\
$\Psi_{X, 12}$ & 14 & $w_{1,11}, w_{2,11}, \cdots, w_{14,11}$ \\
\hline \hline
\end{tabular}

Therefore, in this paper we make use of a GPU computing to pre-compute the light transport instead of the octree. This GPU based computing prevents us from executing the pre-computation repeatedly due to incorrect choice of the solid angle and enables us to produce robust rendering results. After storing the vertex information such as area, location and irradiance into 3 textures, respectively, we evaluate the light transport at a time using a shader program. And the evaluation results are transferred into the system memory through a frame buffer. Similar methods have been used to reproduce the diffusion effect by performing the convolution in texture space using GPUs (d'Eon, et al., 2007). However, since our method takes advantage of the vertices directly for the light transport computation, parameterized meshes are not necessary and we do not have to worry about the distortion of the computed textures during texture mapping.

Because in this paper we use 64 basis, we need a huge storage to contain the pre-computed light transport results for every basis. Ideally, the 64 light transport results need to be separately stored in 64 textures. However, the maximum storage capacity of the texture is limited and the number of textures which can be referred by the shader programs is restricted to 32 in this work. Fortunately, this can be solved by making the use of a texture buffer object (TBO). The texture buffer is a specialized storage for one-dimensional arrays of texels and provides a large storage up to $128 \mathrm{Mbytes}$. We store the 64 light transport data evenly divided into 16 buffers: 4 transport data per buffer, while leaving the rest of buffers as spare ones.

The fewer the number of the basis, the less time it takes to execute the pre-computation and the less storage we need. Therefore, we endeavored to determine the optimal basis set by investigating about 9,000 samples for a broad range of translucent materials. Because this work is based on the diffusion theory, we dealt only with highly scattering materials whose scattering parameters are 10 times larger than absorption. Table 2 lists the wavelet basis used in our method. 


\subsection{Translucent material editing system}

As described in Fig. 1 and in Eq. 8, we can obtain the light flux at each vertex by performing dot product of the precomputed light transports $(T(x o))$ and the wavelet coefficients $(c, w)$ corresponding to each wavelet basis in the vertex shader program. The light flux results are multiplied by the diffuse Fresnel transmittance so as to produce a rendering image. In order to enhance the realism of an object we take the surface reflection into account. In our rendering framework we make full use of the GPU. We exploit the GPU for general-purpose computation (GPGPU) to execute the sampling process for the diffusion profile and the pre-computation for the light transports as well as visualization. This approach provides developers with a new technique to manipulate translucent materials quickly and robustly.

\section{Results and discussion}

All the tests in this work were performed on a personal computer with Intel $2.93 \mathrm{GHz}$ Pentium4 CPU and NVIDIA Geforce GTX560Ti series graphics hardware with $1 \mathrm{~GB}$ video memory. We implemented our proposed algorithm using the OpenGL Shading Language(GLSL). The image resolution of all rendered images in this work is $800 \times 600$.

\subsection{Rendering results}

As can be seen in Fig. 9, although they use the same number of basis, the reconstruction method using low pass filter based Haar wavelet produces an undesirable result while our method provides an accurate rendering result.

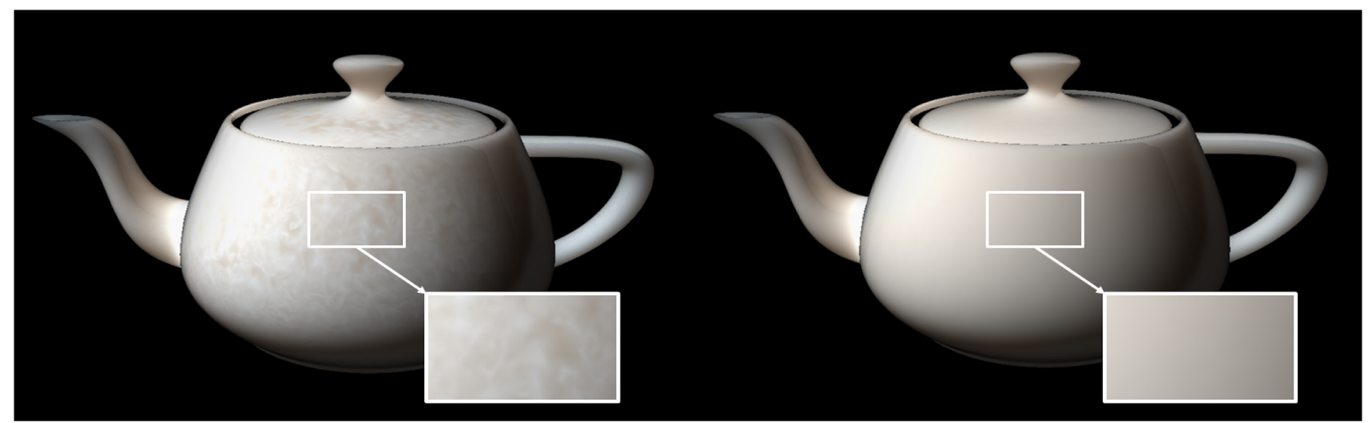

Fig. 9 Accuracy comparison of the approximation results between the LPF based wavelet (left) and the non-linear wavelet (right).

Fig. 10 shows the rendering results with respect o the number of basis. It is can be observed that the rendering image using 64 basis looks almost same as the reference. However, it is worthy to note that in many cases visually convincing results are produced with fewer basis, in which case we can reduce the pre-computation time, but it has little effect on FPS. Conversely, the use of more than 64 basis makes little difference to the rendering accuracy, but unnecessarily increases the computation time and storage.

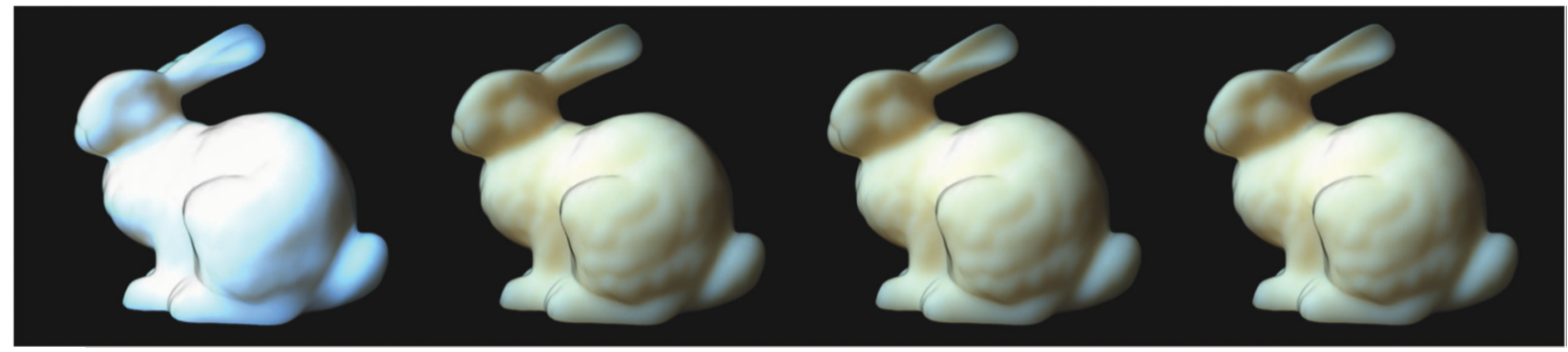

Fig. 10 Rendering results with respect to the number of basis. From left to right, the used basis are 8, 32, 64 and reference. The used materials are skim milk (Jensen, et al., 2001).

The proposed editing algorithm enables the designer to edit all the optical parameters of each sub-layer that includes scattering, absorption, thickness and the refractive index. Fig. 11 and 12 show the combining results of two distinct 
translucent materials with different optical parameters: the same upper layer but different bottom materials (Fig. 11), the same bottom material but different upper layers(Fig. 12).

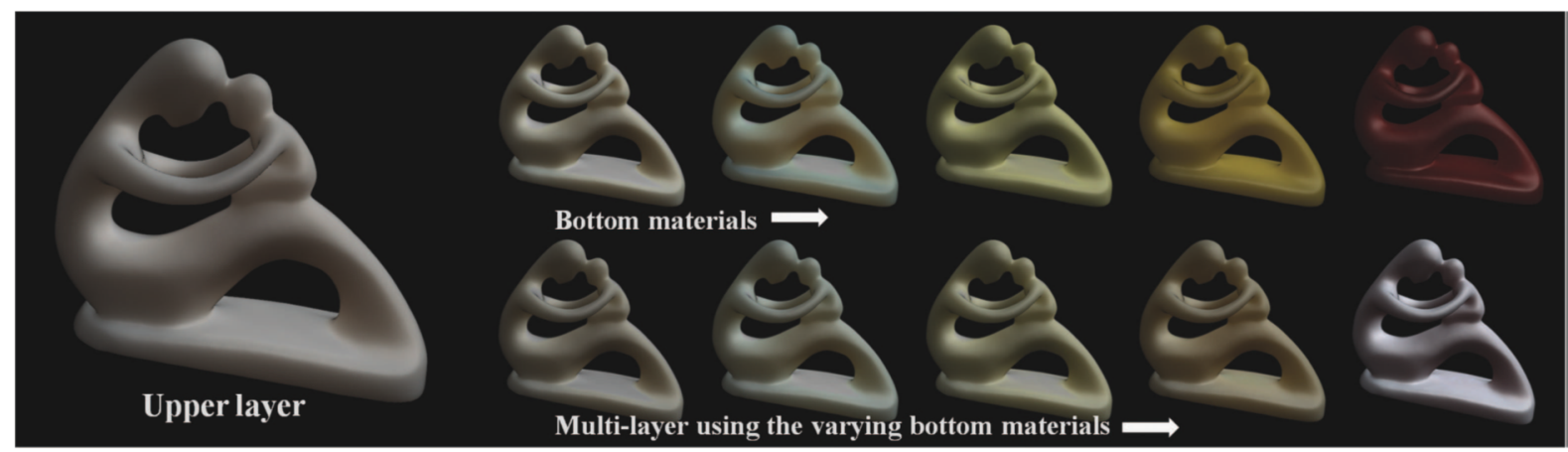

Fig. 11 Rendering results of the multi-layered translucent materials with varying bottom materials. The material of the top layer is fixed(marble), but the bottom materials change(from left to right, whole milk, skim milk, apple, potato and ketchup in (Jensen, et al., 2001), respectively).

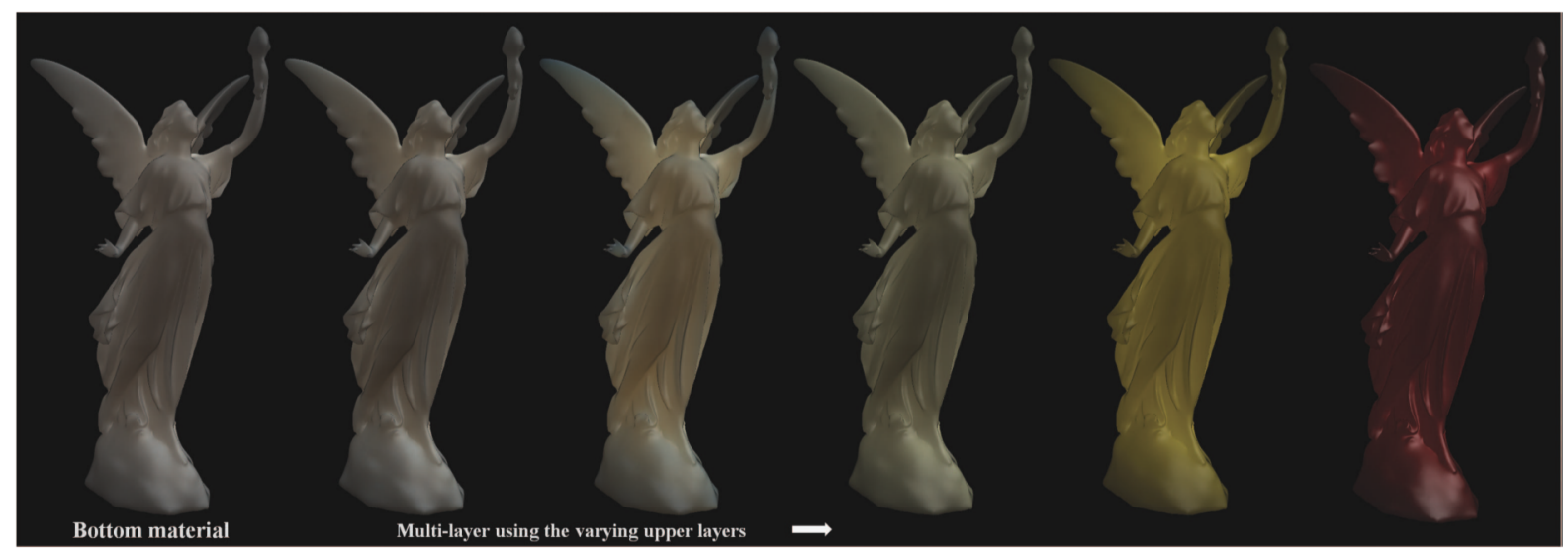

Fig. 12 Rendering results of the multi-layered translucent materials with varying upper layers. The bottom material isfixed(marble), but the upper materials change(the same materials with Fig. 7 are used). For stable results, the thicknesses ofthe upper layers are fixed $2 \mathrm{mfp}$ (mean free path).

\subsection{Performance test}

Main computation for editing materials consists of the convolution computation for diffusion profile generation, wavelet analysis and dot product of the wavelet coefficients and its corresponding light transports. Among them the last two have little effect in the rendering speed unless the number of vertices is huge, but the biggest bottleneck occurs when generating the diffusion profile. Nonetheless, the computation time to generate the diffusion profile of a multilayered translucent material is less than 50ms. Comparing with the previous method (Donner and Jensen, 2006) which takes about 26 seconds, the proposed algorithm is about 500 times faster. Table 3 shows their computational performance in

Table 3 Computation time for generating the diffusion profile of a multi-layered translucent material. This computations time includes 1 di-pole computation $\left(R_{b}^{+}\right)$for the bottom layer, 4 multi-pole $\left(R_{u}^{+}, T_{u}^{-}, T_{u}^{+}, R_{u}^{-}\right)$forthe upper layer and their convolution. sampling resolution is 0.01 about albedo, 0.1 about optical thickness, respectively)

\begin{tabular}{c|ccc}
\hline \hline Miethod & Sampling & Convolution & Total \\
\hline \hline Previous method (Donner and Jensen, 2005) & $0.138 \mathrm{~s}$ & $25.8 \mathrm{~s}$ & $25.9 \mathrm{~s}$ \\
The proposed method & $0.018 \mathrm{~s}$ & $0.030 \mathrm{~s}$ & $0.048 \mathrm{~s}$ \\
\hline \hline
\end{tabular}

detail. Both tests have been performed on the identical computing system. 
Table 4 lists the performance of our algorithm for various 3D models. With the proposed method we can edit single layered translucent materials at 60 frames per second and multi-layered translucent materials at 20 frames per second, respectively. While the naive pre-computation approach needs about 2 hours, the GPU based pre-computation takes about 2.5 minutes for a 3D model with 100,000 vertices. We can also easily control the number of basis since our method makes use of an analytical approach. This is similar to controlling the solid angle when using the octree. In other words, the GPU based pre-computation can be an alternative for algorithms using the octree.

Table 4 Performance comparison for various 3D models (the resolution is set to $800 \times 600$ pixels)

\begin{tabular}{cccccc}
\hline 3D model & Lucy & Fertilty & Bunny & Teapot & Budda \\
\hline No. of Irradiance & $10 \mathrm{~K}$ & $50 \mathrm{~K}$ & $70 \mathrm{~K}$ & $100 \mathrm{~K}$ & $150 \mathrm{~K}$ \\
No. of Basis & 64 & 64 & 32 & 64 & 64 \\
Pre-comp. time(min) & 0.1 & 0.75 & 0.65 & 2.5 & 5.6 \\
Storage(MB) & 23.5 & 57 & 46 & 105 & 170 \\
FPS(single layer) & 60 & 57 & 58 & 57 & 20 \\
FPS(multi layer) & 28 & 19 & 20 & 19 & 15 \\
\hline \hline
\end{tabular}

\section{Conclusions and future work}

In this paper we develop a method to edit multi-layered translucent materials in real-time. To this end, we first obtained the diffusion profiles of each layer and then convolve them to obtain the diffusion profile of the multi-layered translucent material by introducing the QFHT, which provides a fast convolution method while maintaining accuracy. Next, we derive wavelet coefficients for the diffusion profile by applying a non-linear wavelet analysis. Aside from this step, we pre-compute the light transport corresponding to each wavelet basis and store the pre-computation results into texture buffers. Lastly, we render an image at run-time by performing dot product of the wavelet coefficients and the precomputed light transports of each wavelet basis. Under fixed illumination, we can edit single and multi-layered translucent materials at about 60 and 20 frames per second at $800 \times 600$ resolution, respectively. In addition, the proposed method enables us to edit all the optical parameters of individual layers; scattering, absorption, thickness and the refractive index. Because this method is based on an analytical approach, we can easily control the number of basis while maintaining consistent rendering results.

In future work, we plan to improve our editing system working well even in dynamic illumination condition. Furthermore, our algorithm can be used to extend the previous editing method for heterogeneous translucent materials (Ng, et al., 2003)(Song, et al., 2009) to layered heterogeneous translucent materials. In this paper, it is also proved that the nonlinear wavelet analysis is very suitable to approximate diffusion profiles due to its strength in spatial analysis. This observation might lead us to extend our work to other research (Munoz, et al., 2011) that requires an analysis for rapidly decaying curves.

\section{Acknowledgment}

This research is partially supported by Ministry of Culture, Sports and Tourism(MCST) and Korea Creative Content Agency(KOCCA) in the Culture Technology(CT) Research \& Development Program(R2015040004, R2014100088). And it was financially supported by the Ministry of Trade, Industry and Energy(MOTIE) and Korea Institute for Advancement of Technology(KIAT) through the Promoting Regional specialized Industry.

\section{References}

Ben-Artzi A., Overbeck R. and Ramamoorthi R., Real-time brdf editing in complex lighting, ACMTOG 25, 3, (July 2006), pp. 945-954.

Ben-Artzi A., Egan K., Ramamoorthi R. and Durand F., A precomputed polynomial representation for interactive brdf editing with global illumination, ACM TOG 27, 2, (May 2008), 13:1-13:13. 
Colbert M., Pattanaik S. and Krivanek J., Brdfshop: Creating physically correct bidirectional reflectance distribution functions, IEEE Comput. Graph. Appl. 26, 1, (January 2006), pp. 30-36.

D'Eon E., Luebke D. and Enderton E., Efficient rendering of human skin, In Proc. Eurographics symposium on Rendering '07, (2007), pp. 147-157.

D’Eon E. and Irving G., A quantized-diffusion model for rendering translucent materials, ACM TOG 30, 4, (2011), 56:1$56: 14$.

Donner C. and Jensen H. W., Light diffusion in multilayered translucent materials, ACM TOG 24, 3, (July 2005), pp. 1032-1039.

Donner C. and Jensen H. W., Rapid simulation of steadystate spatially resolved reflectance and transmittance profiles of multilayered turbid materials, J. Opt. Soc. Am. A 23, 6, (2006), pp. 1382-1390.

Hanrahan P. and Krueger W., Reflection from layered surfaces due to subsurface scattering, In Proc. SIGGRAPH '93, (1993), pp. 165-174.

Jensen H. W., Marschner S. R., Levoy M. and Hanrahan P., A practical model for subsurface light transport, In Proc. SIGGRAPH '01, (2001) pp. 511-518.

Jensen H. W. and Buhler J., A rapid hierarchical rendering technique for translucent materials, ACM TOG 21, 3, (2002), pp. 576-581.

Kim H. M., Ko K. H. and Lee K. H., Real-time convolution method for generating light diffusion profiles of layered turbid media, J. Opt. Soc. Am. A 28, 6, (2011), pp. 1276-1284.

Kubelka P., New contributions to the optics of intensely light-scattering materials. part ii: Nonhomogeneous layers, J. Opt. Soc. Am. 44, 4, (Apr 1954), pp. 330-334.

Lee S. J., Kim H. M., Seo M. K., Ko K. H. and Lee K. H., Representation of homogeneous translucent materials using the p3 approximation and an image-based bidirectional subsurface scattering reflectance distribution function measurement system, Opt. Eng. 49, 6 (Jun 2010).

Liemert A. and Kienle A., Light diffusion in n-layered turbid media: steady-state domain, J. Biomed. Opt. 15, 2 (Mar 2010).

Munoz A., Echevarria J. I., Seron F. J., Lopez-Moreno J., Glencross M. and Gutierrez D., Bssrdf estimation from single images, Computer Graphics Forum 30, 2, (2011), pp.455-464.

Nicodemus F., Richmond J., Hsia J., Ginsberg I. and Limperis T., Geometrical considerations and nomenclature for reflectance, National Bureau of Standards, Washington, DC. Inst. for Basic Standards (1977).

NG R., Ramamoorthi R. and Hanrahan P., All frequency shadows using non-linear wavelet lighting approximation, ACM TOG 22, 3, (July 2003), pp. 376-381.

Sheng S. C. and Siegman A. E., Nonlinear-optical calculations using fast-transform methods: Second-harmonic generation with depletion and diffraction, Physical Review A 21, 2, (1980), pp. 599-606.

Siegman A. E., Quasi fast hankel transform, Opt. Lett. 1, 1, (Jul 1977), pp. 13-15.

Sloan P. P., Kautz J. and Snyder J., Precomputed radiance transfer for real-time rendering in dynamic, low-frequency lighting environments, ACM TOG 21, 3, (July 2002), pp.527-536

Song Y., Tong X., Pellacini F. and Peers P., Subedit: a representation for editing measured heterogeneous subsurface scattering, ACM TOG 28, 3, (July 2009), 31:1-31:10.

Wang J., Zhao S., Tong X., Lin S., Lin Z., Dong Y., Guo B. and Shum H.-Y., Modeling and rendering of heterogeneous translucent materials using the diffusion equation, ACM TOG 27, 1, 9:1-9:18 (March 2008)

Wang R., Cheslack-Postava E., Wang R., Luebke D., Chen Q., Hua W., Peng Q. and Bao H., Real-time editing and relighting of homogeneous translucent materials, Vis. Comput. 24, 7, (July 2008), pp. 565-575

Xu K., Gao Y., Li Y., Ju T. and Hu S.-M., Real-time homogenous translucent material editing, Computer Graphics Forum 26, 3, (2007), pp. 545-552. 